\title{
Application of International Linear Collider superconducting cavities for acceleration of protons
}

\author{
P. N. Ostroumov* \\ Physics Division, Argonne National Laboratory, 9700 S. Cass Avenue, Argonne, Illinois 60439, USA \\ V. N. Aseev and I. V. Gonin \\ Fermilab, P.O. Box 500, Batavia, Illinois 60510, USA \\ B. Rusnak \\ Lawrence Livermore National Laboratory, 7000 East Avenue, Livermore, California 94550, USA
}

(Received 15 August 2007; published 14 December 2007)

\begin{abstract}
Beam acceleration in the International Linear Collider (ILC) will be provided by 9-cell $1300 \mathrm{MHz}$ superconducting (SC) cavities. The cavities are designed for effective acceleration of charged particles moving with the speed of light and are operated on $\pi$-mode to provide a maximum accelerating gradient. A significant research and development effort has been devoted to develop ILC SC technology and its rf system which resulted in excellent performance of ILC cavities. Therefore, the proposed 8-GeV proton driver in Fermilab is based on ILC cavities above $\sim 1.2 \mathrm{GeV}$. The efficiency of proton beam acceleration by ILC cavities drops fast for lower velocities and it was proposed to develop squeezed ILC-type (S-ILC) cavities operating at $1300 \mathrm{MHz}$ and designed for $\beta_{G}=0.81$, geometrical beta, to accelerate protons or $\mathrm{H}^{-}$from $\sim 420 \mathrm{MeV}$ to $1.2 \mathrm{GeV}$. This paper discusses the possibility of avoiding the development of new $\beta_{G}=0.81$ cavities by operating ILC cavities on $\frac{8}{9} \pi$-mode of standing wave oscillations.
\end{abstract}

PACS numbers: 29.17.+w, 41.20.-q, 29.27.-a, 84.40.-x

\section{INTRODUCTION}

Recently, Fermilab proposed to develop an $\mathrm{H}^{-} 8-\mathrm{GeV}$ linac, or "proton driver" (PD) [1], with the principal mission to raise the intensity of the main injector (MI) to produce so-called superbeams for neutrino studies. There are many other possible applications of the PD linac as are discussed in Refs. [1,2]. The physics design of the most recent PD version was reported elsewhere [3] and includes the following basic concepts.

(i) Directly apply SC elliptical 9-cell cavities and klystrons originally developed for the ILC to accelerate protons above $1.2 \mathrm{GeV}$.

(ii) Develop squeezed ILC-type (S-ILC) cavities operating at $1300 \mathrm{MHz}$ designed for $\beta_{G}=0.81$ to accelerate protons from $\sim 420 \mathrm{MeV}$ to $1.2 \mathrm{GeV}$.

(iii) To simplify the rf system, it is reasonable and cost effective to operate the whole linac at no more than two frequencies. Several options are available for the acceleration of protons up to $\sim 420 \mathrm{MeV}$ at $325 \mathrm{MHz}$ which is a subharmonic of the ILC frequency.

In this paper we present applications of ILC cavities for the acceleration of protons from 600 to $1050 \mathrm{MeV}$ which allows us to avoid the development of S-ILC cavities.

\footnotetext{
*Corresponding author. ostroumov@phy.anl.gov
}

\section{PROPERTIES OF ILC CAVITIES OPERATING ON $\frac{8}{9} \pi$-MODE}

The ILC project requires 14560 9-cell SC cavities [4]. Standard ILC rf units consist of three cryomodules with 26 SC cavities and a quadrupole magnet. As noted in the ILC reference design report [4], "the SCRF cavities and cryomodules are the most technically challenging components and require the largest industrial infrastructure and technical ramp up." The FNAL proton driver requires $\sim 2 \%$ of the total number of ILC cavities and cryostats. Industrial preproduction of ILC cavities can provide a sufficient amount of cavities for the PD. Therefore it is reasonable to provide the largest fraction of the total PD voltage using ILC cryomodules and the rf system without extra development.

The ILC cavity consists of 9 essentially identical accelerating cells and therefore have nine normal modes [5,6]. The frequencies of ILC cavity passbands modes are described by the formula [7]:

$$
f_{q}=\frac{f_{0}}{\sqrt{1+2 k_{\mathrm{cell}}\left[1+\cos \left(\frac{q \pi}{N}\right)\right]}},
$$

where $f_{0}$ is the $\pi$-mode frequency, $q=1,2, \ldots, N, N$ is the total number of cells, and $k_{\text {cell }}=0.010503$. The latter was fitted to follow the measured frequency spectrum [6]. 
TABLE I. Passband frequencies (MHz) of ILC cavities.

\begin{tabular}{cccc}
\hline \hline$q$ & Mode & $\begin{array}{c}\text { Measured } \\
\text { frequency }\end{array}$ & $\begin{array}{c}\text { Frequency calculated } \\
\text { by formula }(1)\end{array}$ \\
\hline 9 & $\pi$ & 1300.091 & 1300.091 \\
8 & $8 \pi / 9$ & 1299.260 & 1299.268 \\
7 & $7 \pi / 9$ & 1296.861 & 1296.908 \\
6 & $6 \pi / 9$ & 1293.345 & 1293.317 \\
5 & $5 \pi / 9$ & 1289.022 & 1288.952 \\
4 & $4 \pi / 9$ & 1284.409 & 1284.355 \\
3 & $3 \pi / 9$ & 1280.206 & 1280.080 \\
2 & $2 \pi / 9$ & 1276.435 & 1276.627 \\
1 & $\pi / 9$ & 1274.387 & 1274.388 \\
\hline \hline
\end{tabular}

The measured and calculated frequencies are given in Table I.

As is seen from Table I, the frequency of the $\frac{8}{9} \pi$-mode is just $\sim 800 \mathrm{kHz}$ lower than the operating mode frequency. The axial electric field distribution for passband modes in an elliptical cell cavity can be described by the simplified formula:

$$
E_{0, q}(z)=-\sin \left(q \pi \frac{2 j(z)-1}{2 N}\right)\left|\sin \left(2 \pi \frac{z}{\lambda}\right)\right|,
$$

where $j(z)=1,2, \ldots, N$ is the integer step function corresponding to the cell number. The field distributions for three adjacent modes are shown in Fig. 1.

Using the formula (2), one can calculate the transit time factor (TTF) for protons. Figure 2 shows TTF calculated for particle velocities from $0.6 c$ to the speed of light, $c$, for three adjacent modes of a 9-cell cavity. The ILC cavities are designed for $31.5 \mathrm{MV} / \mathrm{m}$ accelerating field (see Table 3.6-1 in Ref. [4]) which provides 32.69 MV voltage

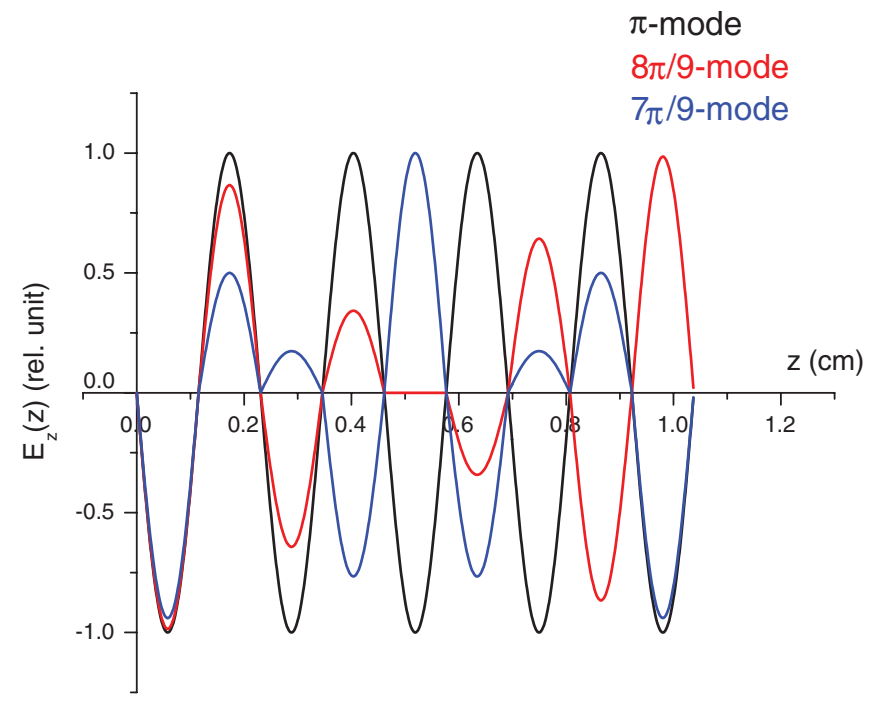

FIG. 1. (Color) Accelerating field distribution in a 9-cell cavity for $\pi-, \frac{8}{9} \pi$-, and $\frac{7}{9} \pi$-modes correspondingly. The fields are calculated by the simplified formula (2).

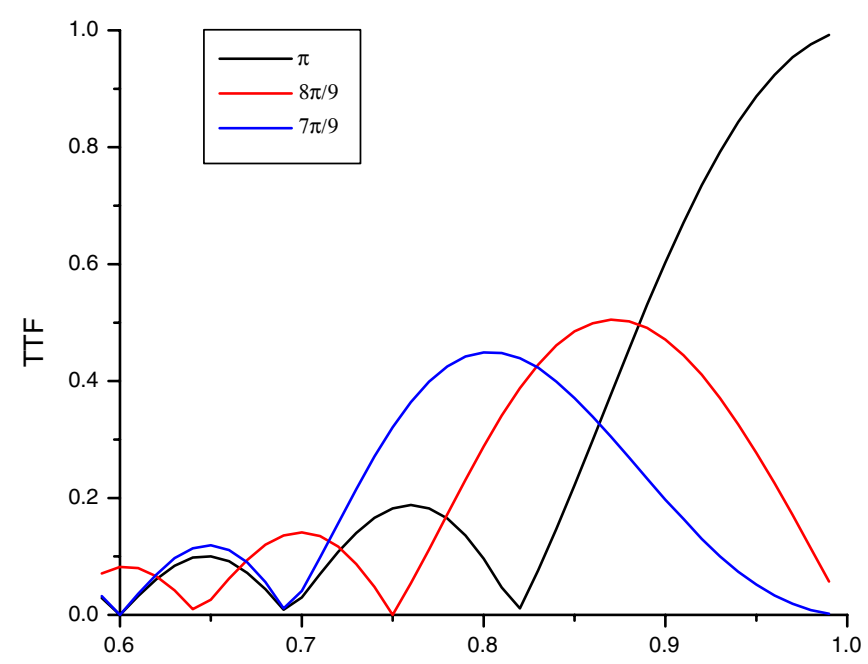

FIG. 2. (Color) Transit time factor of 3 adjacent modes as a function of the particle relative velocity.

gain per cavity. According to the TTF shown in Fig. 2, the acceleration of protons on $\frac{8}{9} \pi$-mode is possible starting from $\beta \approx 0.8$ providing a voltage gain per cavity in the range of 9 to $14.5 \mathrm{MV}$. In fact, the $\frac{7}{9} \pi$-mode can be also used for protons even for lower velocities. However, this mode's frequency is quite far $(\sim 3 \mathrm{MHz})$ from the main $\pi$-mode's frequency and retuning of the cavity cells may require significant deformations before cavity assembly.

We have performed 3D computer simulations to define eigenmodes and field distribution on passband modes of an ILC cavity. The major scope of these simulations is to define the peak electric and magnetic surface fields on $\frac{8}{9} \pi$-mode. The axial electric field distributions on $\pi$ - and $\frac{8}{9} \pi$-modes are shown in Fig. 3. The field distribution was converted to the TRACK [8] format for beam dynamics simulations. We assume the same peak surface fields either electric or magnetic, whichever is the highest, on $\frac{8}{9} \pi$-mode

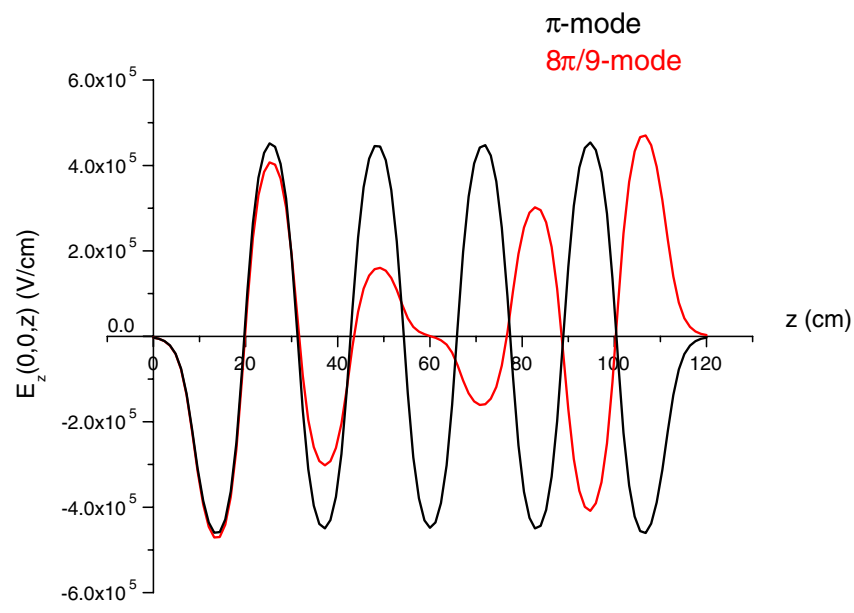

FIG. 3. (Color) Accelerating field distribution along the ILC cavity operating on $\pi$-mode and $8 \pi / 9$-mode. 


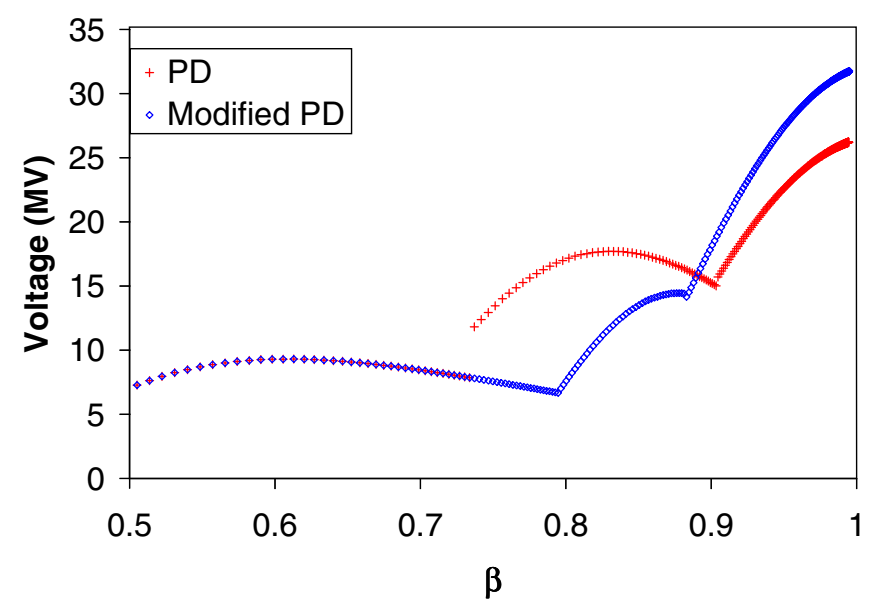

FIG. 4. (Color) Voltage gain per cavity as a function of particle relative velocity. The crosses correspond to the existing PD design reported in Ref. [3], the diamonds show the voltage gain in the modified PD with $8 \pi / 9$-mode cavities.

as on $\pi$-mode. It appears that a peak surface magnetic field is the highest one and is equal to $1340 \mathrm{G}$. Figure 4 shows the proton voltage gain in the section of the PD above $\sim 150 \mathrm{MeV}$ which contains triple spoke resonators (TSR) with $\sim 10 \mathrm{MV} / \mathrm{m}$ accelerating gradient and ILC-type cavities. The main parameters of the linac are shown in Table II. The proposed modifications of the PD parameters with respect to those given in Ref. [3] are the following:

(i) Increase the number of TSRs from 42 to 66 to push the transition energy from $420 \mathrm{MeV}$ to $600 \mathrm{MeV}$.

(ii) Use ILC cavities on $\frac{8}{9} \pi$-mode in the energy range from $600 \mathrm{MeV}$ to $1050 \mathrm{MeV}$. (iii) Provide a peak surface field of $63 \mathrm{MV} / \mathrm{m}$ in ILC cavities of the modified PD design, the same as in the ILC project.

A cavity tuning procedure to implement $\frac{8}{9} \pi$-mode for proton acceleration in ILC cavities includes the following steps: (i) Tuning of cavity cells and a cavity as whole to $\pi$-mode's frequency of $1300.8 \mathrm{MHz}$. Such a tuning may be required for each cell before the cavity assembly because the standard cavity tuner's frequency range is limited to $\pm 300 \mathrm{kHz}[4,5]$. For the frequency control of each cell and tuning of the cavity field "flatness" on $\frac{8}{9} \pi$-mode, standard procedures can be applied $[9,10]$. (ii) Tuning the frequency of the $\frac{8}{9} \pi$-mode to be $1300 \mathrm{MHz}$ in the fully loaded cavity at cryogenic temperature.

In addition, some tuning of a coupler and fast tuner may be required for cavities operating on $\frac{8}{9} \pi$-mode. Specifications to the cavity coupler and fast tuner should be investigated during cavity prototyping.

\section{OPTIMAL TUNING OF CAVITY COUPLERS FOR APPLICATION IN A PROTON LINAC}

To minimize the power requirements, the waveguide-tocavity coupling factor, $\beta_{C}$, must be optimized to define cavity loaded quality factor $Q_{L}$ [11]. In a SC cavity, $Q_{L} \approx$ $Q_{\mathrm{ext}} \approx Q_{0} / \beta_{C}$, where $Q_{\mathrm{ext}}$ and $Q_{0}$ are external and intrinsic quality factors, respectively. A coupler must be positioned to obtain optimal coupling factor:

$$
\begin{aligned}
\beta_{\mathrm{opt}} & \approx \frac{R_{\mathrm{sh}} I_{b} T \cos \varphi_{b}}{V_{0}} \\
\text { which result in } Q_{\mathrm{ext}} & \approx \frac{V_{0}}{R_{\mathrm{sh}} / Q_{0} I_{b} T \cos \varphi_{b}},
\end{aligned}
$$

TABLE II. Main parameters of the existing and modified PD linac designs.

\begin{tabular}{lcc}
\hline \multicolumn{1}{c}{ Parameter } & $\begin{array}{c}\text { Existing } \\
\text { PD design }\end{array}$ & $\begin{array}{c}\text { PD modified } \\
\text { for } \frac{8}{9} \pi \text {-mode option }\end{array}$ \\
\hline Transition energy to ILC frequency $(\mathrm{MeV})$ & 420 & 600 \\
Accelerating gradient in ILC cavities $(\mathrm{MV} / \mathrm{m})$ & 26.0 & 31.5 \\
Number of TSRs & 42 & 66 \\
Number of squeezed ILC cavities & 63 & None \\
Number of ILC cavities operating at $8 \pi / 9$-mode & None & 42 \\
Number of ILC cavities & 287 & 263 \\
Total linac length $(\mathrm{m})$ & 678 & 670 \\
\hline \hline
\end{tabular}

TABLE III. Parameters of ILC cavity for $8 \pi / 9$ - and $\pi$-modes.

\begin{tabular}{lcc}
\hline \hline \multicolumn{1}{c}{ Parameter } & $8 \pi / 9$-mode & $\pi$-mode \\
\hline Optimal velocity providing maximum cavity voltage, $\beta_{G}$ & 0.88 & 1.0 \\
$V_{0}(\mathrm{MV})$ & 14.45 & 32.69 \\
$R_{\mathrm{sh}} / Q_{0}(\mathrm{Ohm})$ & 409 & 1036 \\
Peak surface electric field, $E_{\mathrm{PEAK}},(\mathrm{MV} / \mathrm{m})$ & 60.2 & 63.0 \\
Peak surface magnetic field, $B_{\text {PEAK }}(\mathrm{G})$ & 1340 & 1340 \\
\hline \hline
\end{tabular}




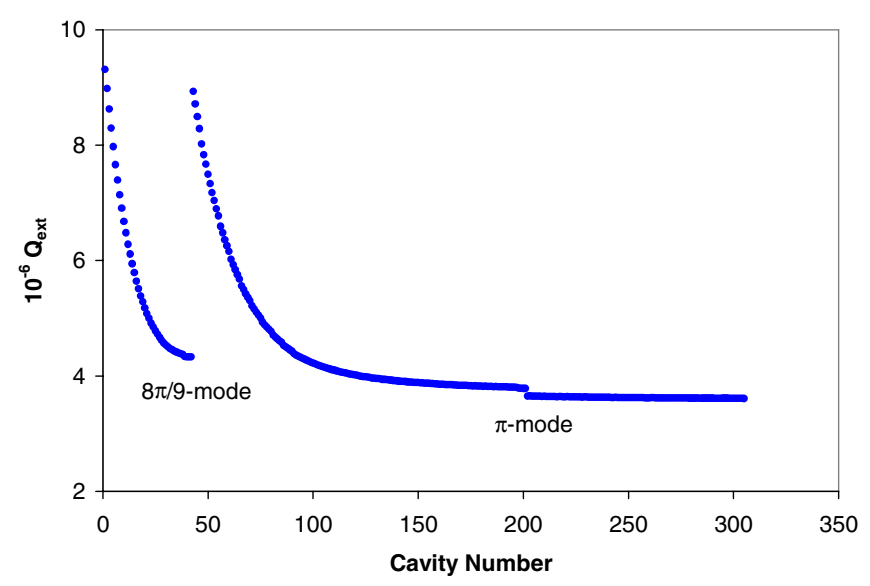

FIG. 5. (Color) $Q_{\text {ext }}$ as a function of cavity number in the high energy section of the modified PD.

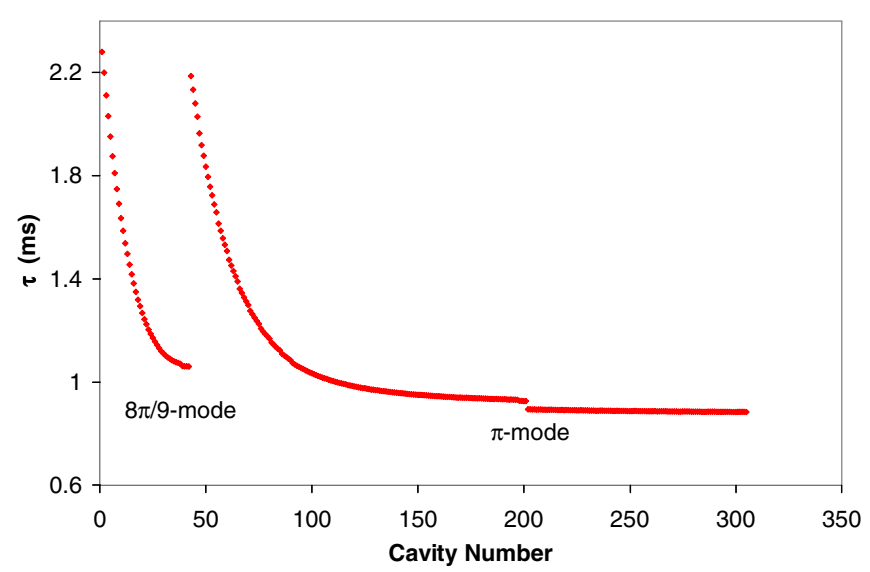

FIG. 6. (Color) The cavity time constant as a function of cavity number in the high energy section of the modified PD.

where $R_{\mathrm{sh}} \approx \frac{V_{0}^{2}}{P_{\text {diss }}}, I_{b}=9 \mathrm{~mA}$ is the average pulse beam current, $V_{0}$ is the highest possible voltage gain of a particle in a cavity, $P_{\text {diss }}$ is the rf power dissipated in the cavity, $\phi_{b}$ is the beam synchronous phase, and $T$ is the transit time factor (TTF). These parameters for ILC cavity operating both on $8 \pi / 9$ - and $\pi$-modes are listed in Table III. The synchronous phase in the high energy section of the PD is varied from $-26^{\circ}$ to $0^{\circ}$. The latter is set for the last 104 cavities (4 rf units).

Because of the variation of the cavity effective voltage and beam synchronous phase along the linac, $Q_{\text {ext }}$ has to be varied to provide minimum rf power to each cavity. Figure 5 shows the required $Q_{\text {ext }}$ as a function of the cavity number. $Q_{\text {ext }}$ defines the cavity time constant as is shown in Fig. 6. As is seen from Fig. 5, the $Q_{\text {ext }}$ is varied from $3 \times$ $10^{6}$ to $9 \times 10^{6}$ which is within the specified range for ILC couplers [5]. The filling time of the first $\sim 10-15$ cavities operating both on $8 \pi / 9$ - and $\pi$-modes can be reduced by slightly detuning the couplers from the regime of minimal rf power consumption.

\section{CONCLUSION}

In the energy range from $\sim 600 \mathrm{MeV}$ to $1050 \mathrm{MeV}$, protons can be accelerated by ILC cavities tuned to operate at $1300 \mathrm{MHz}$ on $8 \pi / 9$-mode. The voltage gain per cavity is higher than in TSRs and it is in the range from 9 to 14.5 MV. The advantage of the proposed concept is using ILC cavities down to $600 \mathrm{MeV}$ and avoiding the development of a new type of cavities for proton linacs. The complete infrastructure of ILC cryomodules, cavities, focusing quadrupoles, and rf system can be applied for the cavities operating on $8 \pi / 9$-mode. This concept can be very efficient for multi-GeV proton or $\mathrm{H}^{-}$machines being considered at FNAL and CERN.

\section{ACKNOWLEDGMENTS}

The authors thank G. Apollinari, D. McGinnis, T. Khabiboulline, and R. Webber (all FNAL) for stimulating discussions. This work was supported by the U.S. Department of Energy, Office of Nuclear Physics, under Contracts No. DE-AC-02-06CH11357 and No. DE-AC0207CH11359. This work was performed under the auspices of the U.S. Department of Energy by the University of California, Lawrence Livermore National Laboratory, under Contract No. W-7405-Eng-48.

[1] G. W. Foster and J.A. MacLachlan, Proceedings of the LINAC-2002, Gyeongju, Korea, p. 826.

[2] $8 \mathrm{GeV}$ Superconducting Injector Linac, edited by G. W. Foster, available at http://tdserver1.fnal.gov/ 8gevlinacPapers/WWWtest/Writeup_v45.doc.

[3] P. N. Ostroumov, New J. Phys. 8, 281 (2006), http://stacks. iop.org/1367-2630/8/281.

[4] ILC RDR, available at http://media.linearcollider.org/ rdr_draft_v1.pdf.

[5] B. Aune et al., Phys. Rev. ST Accel. Beams 3, 092001 (2000).

[6] T. Schilcher, TESLA-Report No. 1998-20.

[7] H. Padamsee, J. Knobloch, and T. Hays, RF Superonductivity for Accelerators (John Wiley and Sons, Inc., New York, 1998).

[8] V.N. Aseev, P.N. Ostroumov, E.S. Lessner, and B. Mustapha, Proceedings of the PAC-2005, Knoxville, TN, p. 2053.

[9] G. R. Kreps, J. Sekutowicz, and D. Proch, TESLA report, 1996, http://dbserv.ihep.su/ pubs/aconf96/ps/c96-123.pdf.

[10] G. Kreps, D. Proch, and J. Sekutowicz, LANL Report No. LA-13782-C, http://laacg.lanl.gov/rfsc99/rfsc99_web/ rfsc99.pdf.

[11] T. P. Wangler, Principles of RF Linear Accelerators (John Wiley and Sons, Inc., New York, 1998). 